\title{
Pasto de corte king grass morado (Pennisetum Purpureum x Pennisetum Typhoides), una esperanza forrajera en la colonia agrícola de Acacias
}

\author{
Purple king grass cut grass (Pennisetum purpureum x Pennisetum Typhoides), the forage \\ hope of the agricultural colony of Acacias \\ Dayro Enrique Cortes Martínez', Oscar Javier Olarte Blandon² \\ ${ }^{1}$ Esp. en Educación Superior a Distancia. ${ }^{2}$ Magister en Administración de Organizaciones. \\ 1,2Docente Escuela de Ciencias Agrícolas, Pecuarias y del Medio Ambiente, Universidad Nacional Abierta y a \\ Distancia - UNAD. \\ 1․ㅣㅁo.cortes@unad.edu.co, ${ }^{2}$ oscar.olarte@unad.edu.co
}

\begin{abstract}
Resumen
El documento contempla una ruta coherente que va desde información esencial (descripción de la especie) como también aspectos relacionados con la semilla, siembra, corte y acarreo, calidad nutricional y aspectos de profundización del pasto de corte king grass morado (Pennisetum Purpureum x Pennisetum Typhoides). Se concluye que el potencial de esta gramínea en la ganadería es importante y que con este elemento forrajero se pueden alcanzar resultados de producción importantes.
\end{abstract}

Palabras clave: King Grass Morado, Pastos de corte, Silvopastoriles.

\section{Introducción}

Los pastos de corte representan una alternativa viable y aplicable a las ganaderías futurista que sueñan con intensificar la producción de carne por área. Los pastos de corte como cultivos forrajeros son una alternativa importante para suplementar al ganado en pastoreo, especialmente en época seca, donde la cantidad y calidad de las praderas se reducen a más del $50 \%$ de su potencial, y como consecuencia de ello se presentan desequilibrios en los parámetros productivos.

Sobre pastos de corte es muy poca la información difundida y la base genética de los forrajes del Piedemonte llanero lo constituyen especies de origen africano incluyendo las gramíneas de ramoneo, entre ellas las del genero brachiaria y de las de corte los del genero pennisetum respectivamente.

En la actualidad los ganaderos se ven abocados a la necesidad de incrementar la capacidad de carga, mejorar la calidad en menor tiempo y a menor costo en las lecherías y la ceba de los animales; esto se puede lograr perfectamente con la implementación de cultivos forrajeros intensivos, ya sea con leguminosas arbustivas (cratylia argentea), especies no convencionales como el botón de oro (Tithonia diversifolia), Morera (morus alba) o con pastos de corte.

Bernal (1991) y Burger (1980), manifiestan que los Pennisetum purpureum son pastos de origen africano, robustos, vigorosos y perennes, por lo cual han sido introducidos en todas las regiones tropicales y subtropicales.

La colonia Agrícola ubicada en la ciudad de Acacias - Meta ve en el pasto Kin Grass Morado un potencial de producción de forraje que puede satisfacer sus requerimientos para la nutrición de sus animales.

Es importante aclarar que el sistema de producción cuenta con otra diversidad de pastos como pasto elefante (Pennisetum purpureum), Maralfalfa (Pennisetum Sp) e Imperial (Axonopus scoparius). Este documento hará referencia a nuestras experiencias sobre el pasto King Grass morado.

DOI: https://doi.org/10.22490/ECAPMA.2772 
Es por esta razón que se ha estructurado un documento producto de la experiencia y enriquecido con consultas bibliográficas en fuentes confiables que soportan un marco teórico que permite conocer y entender el trabajo de alimentación con base en este pasto.

\section{Metodología}

Este documento se inició con la actualización de un trabajo escrito en el 2008 y se reestructuro con búsquedas de fuentes bibliográficas como artículos científicos en google académico y sus amplias publicaciones sobre el pasto Kin grass morado. Como resultado de este estudio se generó una temática en lo referente a generalidades del pasto, metodologías de siembra, fertilización, altura y frecuencia de corte, producción de forraje, calidad nutricional, y sistemas Silvopastoriles. En todas estas consultas se tomaron referentes de la región latinoamericana, libros relacionados, trabajos de grado o comunicaciones de congresos, sin restricción de fecha o tipo de publicación. Esta metodología es similar a la utilizada por Gallego et al 2014.

\section{Resultados y discusión}

\section{Características generales de pasto king grass morado.}

El pasto King grass morado es una variedad hibrida cuyo fundamento genético es el pasto elefante Pennisetum Purpureum, por lo que muchas de sus características morfológicas coinciden. Araya y Boschisini (2005) describen al pasto elefante como una especie perenne, de crecimiento erecto, de 2 a $4 \mathrm{~m}$ de altura, con una caña maciza de 1,5 a $2 \mathrm{~cm}$ de diámetro, hojas lanceoladas, planas, tiernas y algo ásperas de 50 a $100 \mathrm{~cm}$ de largo y de $5 \mathrm{~cm}$ de ancho similares a las del ecotipo morado. Este hibrido tiene origen en la antigua de la republica de Zimbabue en África del Sur. Fue introducido a Suramérica a través de Panamá y a Colombia se trajo en 1.974.

El pasto Rey rojo posee características que lo hacen inconfundible en cuanto a su morfología, sus hojas y tallos poseen un color rojizo o púrpura predominante con relación al verde, más que todo en edad temprana, hasta los 50 días aproximadamente, luego las hojas van tomando un tono un poco más verde, Lemus 2004 citado por Cortes 2007.)

Este pasto, se ha constituido en una de las especies de corte más importantes en los trópicos y subtrópicos por la facilidad que tiene de establecimiento, adaptación amplia a los suelos, buena fertilización, un buen valor nutritivo y además una buena aceptación por el ganado, Gamarra (1985).

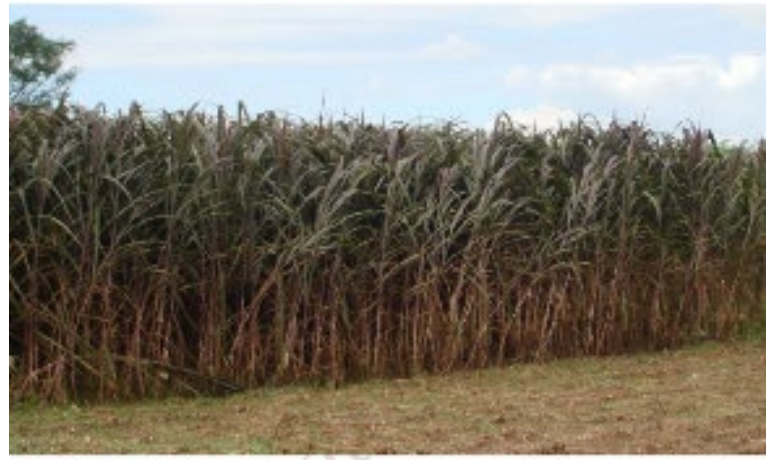

Figura 1. Cultivo de King Grass morado Colonia Agrícola. Fuente: Cortes 2014

\section{Selección del área para el establecimiento de los pastos.}

Como primera medida el cultivo forrajero debe estar cerca de las instalaciones donde se suministra a los animales previo acarreo y fraccionamiento (pica pastos). También se debe tener conocimiento de las características topográficas del terreno; importante que no se presenten encharcamientos, y de ser así construir los drenajes respectivos. De igual manera se debe conocer las características 
en cuanto a fertilidad con análisis de suelos para determinar la aplicación de correctivos y fertilizantes.

\section{Semilla de pasto King Grass morado.}

La semilla botánica de king grass tiene de 10 a $15 \%$ de germinación, aunque se prefiere propagarlo vegetativamente por estacas Rivera (2017).

Se siembra por material asexual (estolones, tallos o cañas) de 3 a 5 yemas, lo que permite obtener rebrotes vigorosos, y lograr un buen establecimiento entre los 120 a 150 días después de la siembra. Se recomienda usar entre 1,5 y 2 toneladas de material vegetativo por hectárea.

Se debe usar semilla que no sea ni muy tierna ni muy vieja; lo mejor es que tenga entre 80 y 90 días de edad para asegurar una buena calidad. Su cantidad depende del sistema de siembra

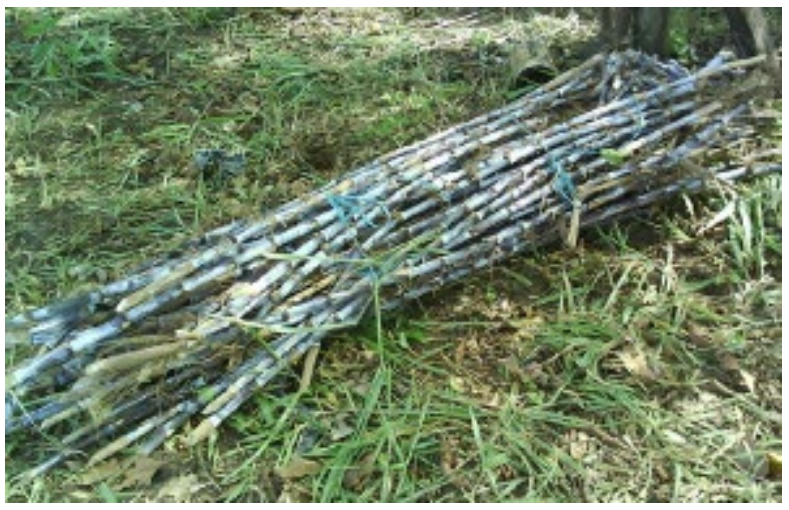

Figura 2. Semilla King Grass morado. Fuente: Cortes 2014

Evaluaciones en este sentido lo realizaron el grupo pecuario de la Colonia agrícola de Acacias en el 2008, en donde en un aforo para determinar la cantidad de semilla requerida para establecer una hectárea con densidades de $0,5 \mathrm{~m}$ entre surcos. Teniendo en cuenta que una hectárea tiene 100 metros de ancho x $100 \mathrm{~m}$ de largo, y que, si tomáramos como referencia estas características del área, tendríamos 200 surcos de 100 metros o el equivalente a 20.000 metros lineales.

El ejercicio consistió en realizar cortes de (estolones, tallos o cañas) semilla de 0,5 metros de largo y pesar un kilogramo de este material. En un kilogramo se contaron 26 estolones.

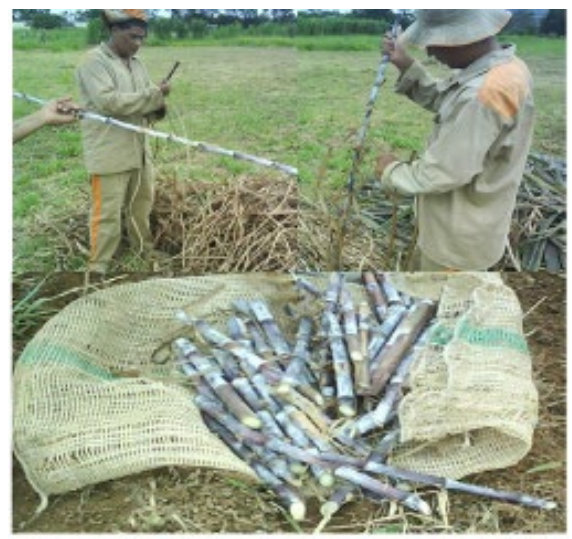

Figura 3. Selección de semilla King Grass morado. Fuente: Cortes 2014 
Si tomamos como referencia el sistema de siembra utilizado en la Colonia que consiste en colocar la semilla vegetativa en hilera donde los tallos se colocan en forma manual con distancias de $0.05 \mathrm{~m}$ entre las puntas. Esto determina que cada segmento de semilla abarca 0,55 m lineales.

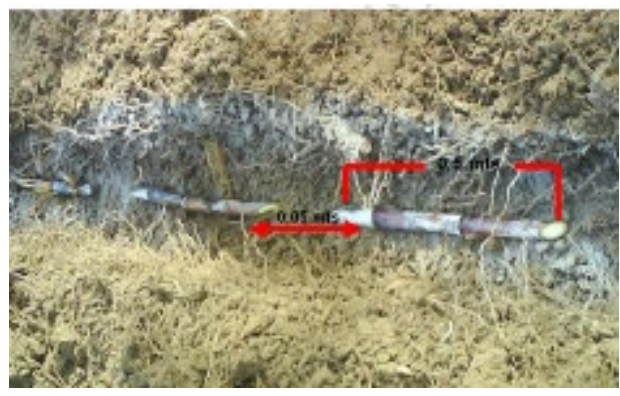

Figura 4. Sistema de siembra. Fuente: Cortes 2014

Si en una hectárea hay que establecer 20.000 metros lineales de pasto (200 surcos de 100 metros) y para establecer 13, 5 metros lineales se requiere de 1 kilogramo de material vegetativo (estolones, tallos o cañas) entonces el material requerido será:

1 kilogramo de semilla $=13,5$. Metros lineales

Para 20.000 Metros lineales que es el equivalente a la siembra de una hectárea el valor de X seria:

$X=$ Kilogramo de semilla $\times 20.000$ metros lineales/ 13,5 metros lineales.

$X=1481$. Kilogramos de semilla/hectárea.

Esta cantidad es el equivalente al sistema de siembra tomado como referente (Colonia Agrícola) y a las condiciones morfológicas de la semilla. Su variación depende de estos dos factores.

\section{Siembra del pasto King Grass Morado.}

Una vez preparado el terreno consistente en un pase de arado de disco longitudinalmente a una profundidad de $25 \mathrm{~cm}$ y dos pases de romplow (El primero longitudinalmente y el segundo transversalmente a una profundidad de $20 \mathrm{~cm}$ ); y habiendo aplicado los correctivos o abonos, de acuerdo con el estado de fertilidad detectado en los resultados de los análisis de suelo se procede a la siembra del material vegetativo.

Es aconsejable realizar una excelente preparación del suelo pues la excesiva mecanización de los suelos afecta sus condiciones físicas tal como lo manifiestan Galvis et al 2016 que argumentan que entre las principales variables de los suelos productivos, la estabilidad estructural y la continuidad del espacio poroso son determinantes por que condicionan físicamente el movimiento de agua, de gases, de nutrientes y desarrollo de raíces, concepción compartida por Salamanca, A., \& Amézquita, (2015) que asegura que la intensidad de uso y manejo del suelo generalmente altera el comportamiento de sus propiedades físicas y acelera su degradación pero que a profundidades de 15 centímetros puede ser positivas de acuerdo a su experiencia en un estudio realizado en suelos del Valle del cauca.

Luego de preparado el suelo se procede a la siembra que inicia con la adición del fertilizante al fondo del surco y colocar la semilla vegetativa en hilera donde los tallos se colocan en forma manual con distancias de $0.05 \mathrm{~m}$ entre las puntas. Esta forma de siembra asegura un rebrote uniforme; cuando el material vegetativo este colocado se procede a taparlo con tierra de los entre surcos. 


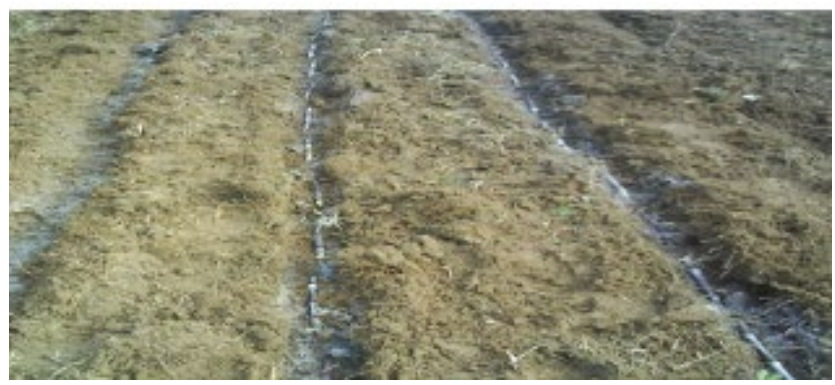

Figura 5. Fertilización y disposición de semilla para la siembra. Fuente: Cortes 2014

La Profundidad de siembra, además de una buena preparación del terreno, para lograr un buen establecimiento, es importante. Por lo tanto, depositar la semilla a una profundidad adecuada contribuye al éxito de un buen establecimiento del cultivo forrajero. Cuando la semilla queda muy superficial, la capa superior del suelo puede alcanzar altas temperaturas, ocasionando deshidratación de las semillas o de las plántulas recién emergidas. En siembras demasiado profundas, las plántulas no alcanzan a emerger, siendo esta una de las principales causas de pérdida de semilla en terrenos sobrepreparados.

Por lo anteriormente expuesto los tallos se deben cubrir totalmente con una capa de suelo no superior a los 5 centímetros, estableciendo íntimo contacto con el material.

A los 7 días de sembrado el material vegetativo (estolones, tallos o cañas), emergen los hijuelos, estos presentan una altura de $10 \mathrm{~cm}$, al mes $75 \mathrm{~cm}$ y a los 4 meses 3 metros, indicando una tasa de crecimiento de $2,5 \mathrm{~cm} /$ día.

Rojas (2009) recomienda la Siembra Inclinada que consiste en que una vez preparado el terreno y cortada la semilla en trozos que tengan por lo menos tres yemas, se entierran las estacas o trozos en forma inclinada, dejando una yema afuera y separadas $50 \times 50 \mathrm{~cm}$.

Otros estudios con respecto al sistema de siembra recomiendan que esta debe ser a 45 grados de inclinación, en otros estudios Leonard et al (2015) compararon dos sistemas de siembra (método 1 estacas acostadas cero grados y método 2 a 45 grados de inclinación) las estacas seleccionadas para este estudio, presentaban nudos y fueron colocadas a $5 \mathrm{~cm}$ de profundidad, a $25 \mathrm{~cm}$ de distancias entre estacas y a $70 \mathrm{~cm}$ entre calles. A los 19 días de edad se midió la germinación, se observaron diferencias en el porcentaje donde el método inclinado presento el mayor porcentaje $100 \%$ y las parcelas de siembra acostada con porcentajes de germinación del 96\%, al cosechar ambas parcelas resulto que la siembra inclinada rindió 9,23 t/h de materia seca contra 5,06 para la siembra acostada.

Otro método es el recomendado por Flores 1986 citado por Leonard et al (2015) denominado por cañas comprende el tallo entero, despuntado desprovisto de hojas de la gramínea; se tira la caña al fondo del surco y se tapa con tierra. En la parte aérea de cada nudo emitirá nuevas plantas y en la interna, raíces.

Por otro lado, Ramos et al., 1979., citados por Leonard et al (2015) aconsejo utilizar material vegetativo de la parte central del tallo, obteniendo mejores resultados en cuanto a calidad y germinación.

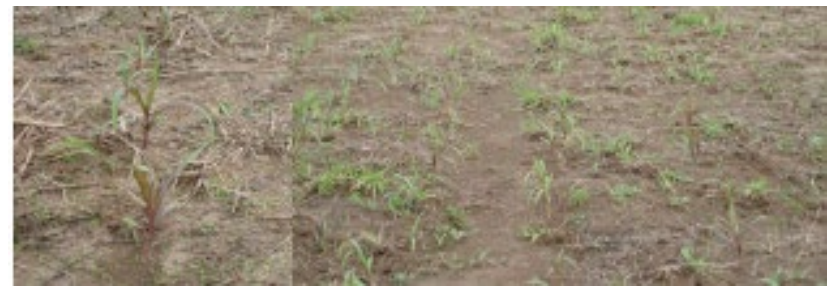

Figura 6. Altura de $10 \mathrm{~cm}$, al emerger los hijuelos de King grass. Fuente: Cortes 2014 


\section{Resiembra}

Consiste en volver a sembrar aquellos sitios en donde se ha perdido la macolla de pasto. Es recomendable hacerlo luego de la cosecha. Probablemente a este tema no se le dé la importancia que merece, pero si no se resiembra, se les da más oportunidad a las arvenses, y se obtiene menor producción de biomasa de pasto.

Es decir, todo depende de la cantidad de vacío o espacios sin pasto en el cultivo, los cuales no deberían existir.

\section{Altura y frecuencia de Corte}

La altura a la cosecha está muy relacionada con el tiempo de crecimiento después del corte e influye sobre la calidad y el rendimiento, pero el primer aspecto, es el que afecta directamente la respuesta animal, especialmente en el consumo y en la producción, así como, en el vigor del futuro rebrote.

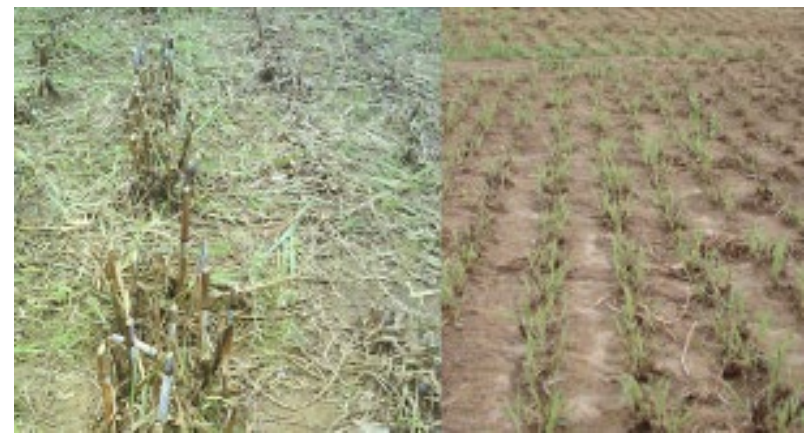

Figura 7. Corte de King Grass. Fuente: Cortes 2014

Para el pasto elefante (Pennisetum purpureum) y king grass, el corte debe ser lo más cerca del suelo, a unos $5 \circ 10 \mathrm{~cm}$. Todo depende de la ubicación de las reservas para el rebrote. También es importante que las herramientas utilizadas (machetes) tengan buen filo y que el personal que está a cargo de esta actividad este entrenada; de esa forma se evitarán daños a las macollas donde provienen los rebrotes, los cuales son importantes para un alto rendimiento.

Los cortes deben realizarse cada 35 a 45 días en época de lluvias y hasta cada 60 días en verano o cuando el pasto alcance una altura de 1.20 a 1.50 con corte al ras del suelo.

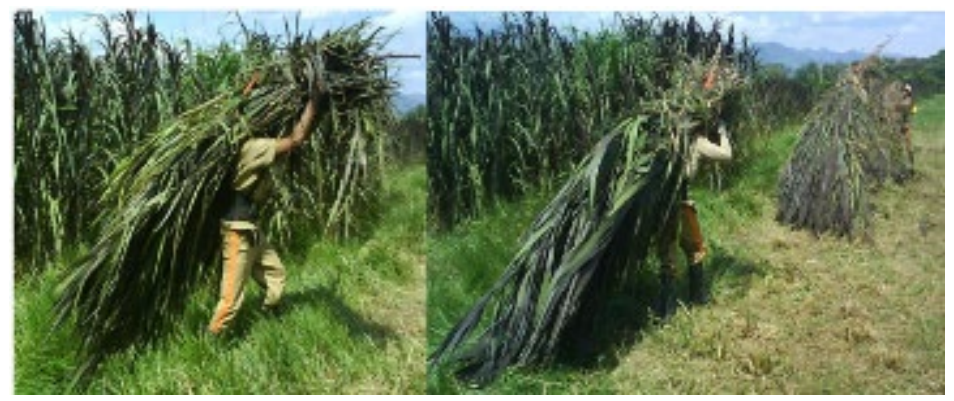

Figura 8. Acarreo de pasto King Grass. Fuente: Cortes 2014

Se recomienda cortar el pasto en las horas de la tarde, después de que la planta haya realizado la fotosíntesis y acumulados carbohidratos solubles (almidón), los cuales están depositados en hojas y tallos. También el contenido de humedad ha disminuido por el aumento de la transpiración durante 
el día, facilitando el acarreo, picado y posiblemente una mayor digestibilidad de la materia seca Dávila y Urbano 2005.

En el caso de los pastos elefante, king grass y la misma caña forrajera, que cuentan con tallos gruesos deben picarse entre 1 a 2,5 cm de largo.

\section{Fertilización}

De esta práctica depende fundamentalmente el éxito de la actividad forrajera, ya que el corte remueve la totalidad de los nutrientes de la parte aérea, los cuales, al ser extraídos del suelo, algunos se tornarán limitantes al crecimiento del pasto. Esto explicaría las caídas bruscas de producción, generalmente a partir del segundo año. Aspecto que equivocadamente se le atribuye al material forrajero.

Según Dávila y Urbano 2005 la práctica difundida de aplicar nitrógeno una semana después del corte y anualmente un fertilizante completo que reponga lo extraído por el cultivo, garantiza la estabilidad del corte.

La fertilización debe ser una herramienta que ayuda a balancear la producción de forraje. Se debería abonar a salida de lluvias, para incrementar la oferta en el verano y bajar la cantidad de fertilizantes durante los periodos de rápido crecimiento, en los cuales el exceso de forraje producido se desperdicia o se madura perdiendo calidad.

Es importante considerar la aplicación de materia orgánica (abonos orgánicos) producidos en la finca con subproductos de la ganadería (bosta). Galviz et al 2016 manifiesta que la presencia de materia orgánica favorece la agregación de las partículas del suelo en agregados de mayor tamaño y repercute en la porosidad de éste permitiendo el avance de las raíces y el movimiento del aire y del agua.

A la vez es necesario establecer un compromiso de seguir incursionado en la apropiación, aplicación y validación de saberes rurales que se han venido perdiendo por la modernidad de la agricultura para que sean adoptados de manera eficiente en la producción de pasto de corte; fertilizaciones alternativas orgánicas estrategia aplicada con las Escuelas Campesinas de Agroecología (ECAS) que surgieron en el municipio de Tuluá a comienzos del año dos mil. Álvarez et al 2013. De esta manera se empezaría a construir indicadores de sustentabilidad que nos alejen del uso indiscriminado de agrotóxicos y una reconciliación con la agroecología para la producción ganadera experiencia exitosa de Mendoza et al 2015 que mediante la IAP (Investigación Acción Participante) promovió la reflexión de producir y comercializar de manera orgánica y con el uso de recursos propios y no de externalidades; estas últimas son las responsables de los cambios del clima y la contaminación que debe ser frenada desde adentro (desde la finca, desde la forma de producir).

\section{Producción de forraje}

EI CIAT (2003) en evaluaciones realizadas por ganaderos del Valle del Cauca, bajo condiciones de fertilidad y humedad adecuadas, obtuvo una producción de 50 - $70 \mathrm{t}$ de forraje verde/ corte, equivalente a 10-14 t/ha de materia seca mediante cortes cada 45-60 días. Bernal 1997 coincide con este nivel de producción y además dice que se pueden lograr seis a ocho cortes al año con una producción de 300 a 400 toneladas de forraje verde lo cual equivale a una producción de 60 a 80 t/ha/año de forraje seco

Arenas (2011), menciona que el crecimiento de raíces y su función depende de la energía proporcionada por la fotosíntesis y correcta nutrición de los cultivos, nos llevara directamente a lograr una buena producción de forrajes.

Según Araya \& Boschini (2005), el pasto King Grass Morado o pasto Camerún como es llamado en Costa Rica debe ser cortado alrededor de los $70-84$ días después del rebrote, edades superiores afectan la producción ya que aumenta la proporción de tallo - hoja y en consecuencia su eficiencia.

Molina (2005), evaluando el comportamiento agronómico del pasto Maralfalfa, logró determinar que el porcentaje de MS se incrementó a medida que aumentaba la edad, con porcentajes promedios de 
$13,8,18,5$ y $24,4 \%$ a los 35,45 y 60 días, respectivamente. Esto indica que el comportamiento en términos de materia seca aumenta y que es necesario tener en cuenta la relación hoja tallo ya que esto afecta en términos de fracciones de la planta que tienen que ver con el consumo y calidad nutricional.

Ramos et al 2013 encontraron que el King grass en marcos de siembra de 0,5 metros entre plantas

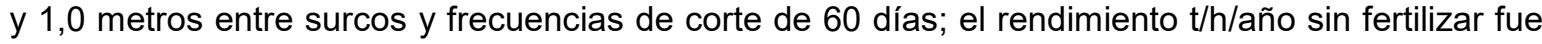
de 102,7 t/h/año, y con fertilizaciones de $300 \mathrm{~kg} / \mathrm{ha} / \mathrm{año}$ de urea y Agua Residual Porcina los rendimientos t/h/año fueron de 131,86 y 140,00 respectivamente.

Madera et al 2013 reportaron que el pasto King grass morado a los 120, 105 y 90 días, en el largo y ancho de la hoja del pasto presentaron valores de $115.50,113.75$ y $102.00 \mathrm{~cm}$ y $5.38,5.10$ y 5.15 $\mathrm{cm}$ respectivamente.

\section{Calidad nutricional.}

Roncallo et al 2012 encontraron en rebrotes de 3,6 y 9 semanas porcentajes de proteína de $20 \%$, $9,5 \%$ y $8,5 \%$ respectivamente.

Vela, (1992). Obtuvo en sus estudios un contenido promedio de proteína cruda (PC) es $8.3 \%$, variando entre 4.7 y $5.3 \%$ en los tallos, a 8.8 y $9.5 \%$ en las hojas. La fertilidad del suelo y la edad de la planta determinan la composición química del forraje

Cartago (2008) mencionado por Chacón y Vargas 2009 en análisis de calidad nutricional del pasto King grass morado en frecuencias de corte de 65,75 y 90 días encontraron porcentajes de Materia seca de $13.03,13.79$ y 14.43 y proteína cruda de $9.56,8.70$ y 9.56 respectivamente.

Por otro lado, López en el 2015 en su estudio análisis bromatológico de pasto morado (pennisetum purpureum) a seis intervalos de corte $45,60,75,90,105$ y 120 días (planta completa), reporto porcentajes de Materia seca de 16.1, 30.3, 17.5, 14.9, 13.0 y 13,5 y proteína cruda de 10.8, 10.5, $6.5,9.1,7.9$ y 6.6 en esos intervalos de corte respectivamente.

\section{Conclusiones}

Con pasto King Grass morado la oferta de forraje por hectárea con densidades de 0,5 metros entre surcos, por ciclo (60 días en invierno - 75 - 85 en verano) la producción de materia verde/hectárea - ciclo, es de 20 toneladas o el equivalente a 4 toneladas de materia seca/hectárea - ciclo esto indica una oferta importante de producción de forraje por área.

De igual manera el aumento y fortalecimiento progresivo de las áreas destinadas a cultivos forrajeros tienden a llegar a dar inicio a una ganadería semintensiva (semiestabulacion).

El pasto King grass morado representa un material que ha demostrado en el tiempo un potencial que satisface tanto en volumen, calidad nutricional y contribución al desarrollo productivo de la ganadería.

De igual manera en este documento se presenta de manera detallada información que es relevante para conocer más de cerca la materia prima (pasto kin grass) para entenderlo y manejarlo.

\section{Literatura citada}

Álvarez Ramírez, F., Castaño Arcila, G., Montes Moreno, J., \& Valencia Trujillo, F. (2013). Las escuelas campesinas de agroecología, centros de formación campesina y los custodios de semillas en los Andes tulueños (Colombia). Revista de Investigación Agraria y Ambiental, 4(2), 135-147. doi:https://doi.org/10.22490/21456453.988 
Araya Mora, M., \& Boschini Figueroa, C. (2005). Producción de forraje y calidad nutricional de variedades de Pennisetum purpureum en la Meseta Central de Costa Rica. Agronomía Mesoamericana, 16(1).

Arenas, D. (2011). Manual de fertilización, manejo de forrajes y pastos cultivados. Colombia. 56 p.

Bernal, J.E. (1991). Pastos y forrajes tropicales. Producción y manejo. Unidad de Divulgación y Prensa. Banco Ganadero. $2^{\mathrm{a}}$ Ed. Bogotá, Colombia. 544 p.

Burger, W. (1980). Flora costarricensis. Publisher by Fiel Museum of Natural History. 4th Edition. United Estates of America. 608 p.

Chacón, H. y Vargas, C. (2009). Digestibilidad y calidad del Pennisetum purpureum cv. King grass a tres edades de rebrote. [En línea]: http://www.ucol.mx/revaia/portal/pdf/2013/mayo/3.pdf.

CIAT, (2003). Manual para la Evaluación Agronómica. Red Internacional de Evaluación de pastos tropicales. Editor técnico: José M. Toledo Cali. Colombia.170 p

Cortes, D. E. (2014). SlideShare. Obtenido de Pasto king grass morado: https://es.slideshare.net/dayroenriquecortesmartinez/pasto-king-grass-morado

Cortes, D.E. (2007). Especies forrajeras para la alimentación de bovinos, aplicado a la colonia agrícola. Acacias, Colombia.106 p.

Davila, C.; y Urbano, D. (2005). Manual de Ganadería Doble Propósito. Uso de pastos de corte en los sistemas intensivos. Merida, Venezuela. Universidad de Los Andes. Instituto de Investigaciones Agropecuarias (ULA-IIAP). pag 193-198.

Gallego, L. A., Mahecha-Ledesma, L., y Angulo-Arizala, J. (2014). Potencial forrajero de Tithonia diversifolia Hemsl. A Gray en la producción de vacas lecheras. Agronomía Mesoamericana, 25(2)

Galvis Quintero, J., Chaparro Anaya, O., Bernal Riobo, J., \& Baquero, J. (2016). Evaluación de la estabilidad estructural y espacio poroso en un Oxisol de sabana de los Llanos Orientales de Colombia. Revista de Investigación Agraria y Ambiental, 7(1). doi:https://doi.org/10.22490/21456453.1613

Gamarra, J.C. (1985). Pennisetum purpureum: su productividad agronómica y valor nutritive en la zona henequenera de Yucatán y su uso en la alimentación animal. Tesis de Maestría en ciencias. Facultad de Medicina Veterinaria y Zootecnia, Universidad Autónoma de Yucatán, México. 124.

Hernández, D., Carballo, M., y Reyes, F. (2000). Reflexiones sobre el uso de los pastos en la producción sostenible de leche y carne de res en el trópico. Pastos y Forrajes, 23(4).

Leonard, I., Burgos, C. V., Uvidia, H., Torres, V., Andino, M., y Benítez, D. (2015). Influencia del método de siembra sobre la curva de crecimiento del Pennisetum purpureum vc King grass en la Amazonía Ecuatoriana. Revista Amazónica Ciencia y Tecnología, 3(1), 33-48.

López R, R. (2015). Análisis bromatológico de pasto morado (Pennisetum purpureum) a diferentes intervalos de corte. Universidad Autónoma Agraria - Antonio Narro - México. 43

Madera, N. B., Ortiz, B., Bacab, H. M., y Magaña, H. (2013). Influencia de la edad de corte del pasto morado (Pennisetum purpureum) en la producción y digestibilidad in vitro de la materia seca. Avances en Investigación Agropecuaria, 17(2).

Mendoza Velásquez, S., Cano Muñoz, J., \& Rojas Sánchez, F. (2015). Acción comunitaria frente al fenómeno del cambio climático, en el páramo de la región del Guavio, Cundinamarca, Colombia. Revista de Investigación Agraria y Ambiental, 6(1), 265 - 279.

doi:https://doi.org/10.22490/21456453.1286 
Mojica, R. J., Castro, R, E., Carulla, F.J., y Lascano, A. C. (2017). Efecto de la edad de rebrote sobre el perfil de ácidos grasos en gramíneas tropicales. Corpoica. Ciencia y Tecnología Agropecuaria, 18(2), 217-232.

Molina S. 2005. Evaluación agronómica y bromatológica del pasto Maralfalfa (Pennisetum sp.) cultivado en el Valle del Sinú. Rev. Fac. Nac. Agron. Colombia. 58(1): 39.

Murgueitio, E. (2003). Investigación participativa en sistemas silvopastoriles integrados: La experiencia de CIPAV en Colombia. Taller Internacional Ganadería Desarrollo Sostenible y Medio Ambiente. La Habana, Cuba. p. 207

Navas, P.A. (2007). Sistemas silvopastoriles para el diseño de fincas ganaderas sostenibles. Revista ACOVEZ, (16), 3-5.

Ramos, T.O., Canul, S. J., y Duarte, V. F. (2013). Producción de tres variedades de Pennisetum purpureum fertilizadas con dos diferentes fuentes nitrogenadas en Yucatán, México. Revista Bio Ciencias, 2(2).

Rivera, R. R. (2017). Evaluación de dos sistemas y cuatro distancias de siembra del pasto King grass morado (Pennisetum purpureum), en la zona de Babahoyo, provincia de Los Ríos (Bachelor's thesis, Babahoyo: UTB, 2017).

Rojas S., (2009). Análisis bromatológico pasto elefante morado (Pennisetum purpureum) Disponible en:http://buendato.com/profiles/blogs/analisis-bromatologico-pasto

Roncallo, B., Milena Sierra, A., \& Castro, E. (2012). Rendimiento de forraje de gramíneas de corte y efecto sobre calidad composicional y producción de leche en el Caribe seco. Corpoica. Ciencia y Tecnología Agropecuaria, 13(1)

Salamanca Jiménez, A., \& Amézquita Collazos, E. (2015). Influencia de la intensidad de uso sobre algunas propiedades físicas en un suelo del Valle del Cauca, Colombia. Revista de Investigación Agraria y Ambiental, 6(1), 43 - 52. doi:https://doi.org/10.22490/21456453.1262

Sánchez, M. D., y Méndez, R. (1999). Agroforestería para la producción animal en América Latina (No. 631.588 C65).

Vela, F. (1992). Dosis de fertilización y distancia de Siembra en el pasto King Grass kin grass (Pennisetum purpureum x P. typhoides) en el cantón Santo Domingo de los Colorados. Tesis de Ing. Agr. Quito: Universidad Central del Ecuador, Facultad de Ciencias Agrícolas. 